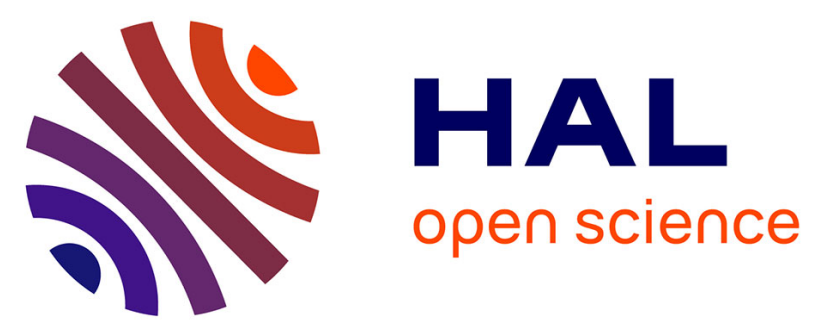

\title{
Tuning of internal gain, dark current and cutoff wavelength of UV photodetectors using quasi-alloy of BGaN-GaN and BGaN-AlN superlattices
}

Jean-Paul Salvestrini, A. Ahaitouf, H. Srour, S. Gautier, T. Moudakir, B. Assouar, A. Ougazzaden

\section{To cite this version:}

Jean-Paul Salvestrini, A. Ahaitouf, H. Srour, S. Gautier, T. Moudakir, et al.. Tuning of internal gain, dark current and cutoff wavelength of UV photodetectors using quasi-alloy of BGaN-GaN and BGaN-AlN superlattices. SPIE Photonic West, Jan 2012, San Francisco, United States. pp.82682S, 10.1117/12.914800 . hal-00642175

\section{HAL Id: hal-00642175 \\ https://hal.science/hal-00642175}

Submitted on 31 Jan 2022

HAL is a multi-disciplinary open access archive for the deposit and dissemination of scientific research documents, whether they are published or not. The documents may come from teaching and research institutions in France or abroad, or from public or private research centers.
L'archive ouverte pluridisciplinaire HAL, est destinée au dépôt et à la diffusion de documents scientifiques de niveau recherche, publiés ou non, émanant des établissements d'enseignement et de recherche français ou étrangers, des laboratoires publics ou privés.

\section{(ㄷ)(1) $\$$}

Distributed under a Creative Commons Attribution - NonCommerciall 4.0 International 


\title{
Tuning of internal gain, dark current and cutoff wavelength of UV photodetectors using quasi-alloy of BGaN-GaN and BGaN-AlN superlattices
}

\author{
Salvestrini J.P. ${ }^{a, b}$, Ahaitouf A. ${ }^{b, d}$, Srour H. ${ }^{a}$, Gautier S. ${ }^{a, b}$, Moudakir T. ${ }^{b}$, Assouar B. ${ }^{b, c}$, and \\ Ougazzaden A. ${ }^{b}$, \\ ${ }^{a}$ Laboratoire Matériaux Optiques, Photonique et Systèmes, EA 4423, Université de Lorraine et \\ Supelec, 2 rue E. Belin, 57070 Metz, France \\ ${ }^{b}$ Georgia Institute of Technology / GT-Lorraine-UMI 2958, Georgia Tech-CNRS, 2-3 rue \\ Marconi, 57070 Metz, France; \\ ${ }^{c}$ Institut Jean Lamour, UMR CNRS 7198, Faculte des Sciences et Technologies, Campus \\ Victor Grignard, 54506 Vandoeuvre-les-Nancy, France \\ ${ }^{d}$ Faculte des Sciences et Technologies, Sidi Mohammed ben Abdellah University, Fès, Morocco
}

\begin{abstract}
Metal-semiconductor-metal solar blind ultraviolet photodetectors have been fabricated using both BGaN-GaN and BGaN-AlN superlattices as active layers. A high internal gain (up to $3 \times 10^{4}$ for optical power in the $\mathrm{nW}$ range) is obtained with a highly reduced dark current thanks to the boron incorporation. In the high optical power regime ( $\mathrm{W}$ range), the time response is in the nanosecond range, which is much smaller than that of GaNand $\mathrm{ZnO}$-based ultraviolet photodetectors. Moreover, the boron incorporation in GaN material allows the tuning of the cutoff wavelength.
\end{abstract}

Keywords: UV photodetector, BGaN, GaN, Superlattices, Schottky

\section{INTRODUCTION}

Gallium nitride (GaN) and its alloys including AlGaN, InGaN, BGaN, and BAlGaN are excellent candidates for semiconductor based photodetectors (PDs) of ultraviolet (UV) radiations. This ability is mainly due to the widely adjustable direct band gap energies in those materials. As seen in Fig.1, the band gaps range start from $0.7 \mathrm{eV}(\mathrm{InN})$ up to $6.2 \mathrm{eV}(\mathrm{AlN})$, suitable for band-to-band light generation or detection with colors ranging from red to ultraviolet wavelengths.

The rationale behind the development of such detectors lies in their wide number of potential applications, including flames sensing, gas detection, missile warning, space and ozone layer monitoring, UV astronomy, water purification, plant and vegetation growth monitoring, submarine communication and medical applications. $^{1,2}$ Moreover III-Nitride compounds can be easily integrated in integrated electronic systems including current amplifiers and electronic gain controls, leading to easily transportable systems. Recent researches reported successful UV PDs engineering on various materials such as III-N ${ }^{3-8}$ and MgZnO alloys. ${ }^{1,9-14}$ Different structures, such as photoconductive, ${ }^{5,13} \mathrm{p}$-i-n, ${ }^{12,14}$ Schottky barrier, ${ }^{4,6,7,11,12}$ and metal-semiconductor-metal $(\mathrm{MSM})^{1,3,7,10}$ were used in the fabrication of these PDs. Some of them exhibit extraordinary values of the responsivity $(\mathrm{R})$ corresponding to an apparent external quantum efficiency (QE) exceeding 100\%, typically in the range of 2 to $9 \times 10^{4}, 1,3,5,9-14$ indicating large internal gains in these PDs. These unexpected high values of the internal gain have been explained by several physical mechanisms. Most of the authors ${ }^{1,3-5,7,9,10,15-17}$ attribute this gain phenomenon to the existence of trapping states at the active layer-metal interface. In GaNtype materials for example, these traps are generally attributed to threading dislocations, having acceptor-like

Send correspondence to J.P. Salvestrini: E-mail: salvestr@metz.supelec.fr, Telephone: +33 (3) 87378567 


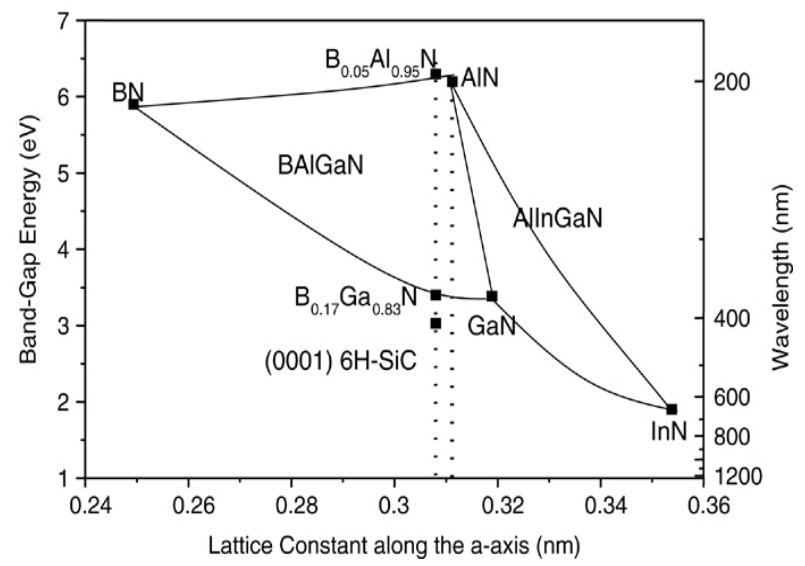

Figure 1. Band gap energy at $\mathrm{T}=300 \mathrm{~K}$ versus lattice constant in III-N semiconductors

nature. ${ }^{3}$ Under illumination, these interface states can trap photo-generated holes, leading to a Schottky barrier height $(\mathrm{SBH})$ decrease $\Delta \Phi_{b}$ and producing a secondary photocurrent ${ }^{15} I_{\lambda}^{G}$ :

$$
I_{\lambda}^{G}=I_{d} e^{\frac{\Delta \Phi_{b}}{k T}}
$$

where $I_{d}$ is the dark current in the structure, leading to a gain in the PD. This secondary current appears in addition to the "true" photocurrent generated by the photodetector given by:

$$
I_{\lambda}=q \times S \times P\left(1-\exp \left(-\alpha \sqrt{\frac{2 \epsilon\left(V_{b i}-V\right)}{q N_{D}}}\right)\right)
$$

where $\mathrm{q}$ is the electronic charge, $\mathrm{S}$ is the area of the contact, $\mathrm{P}$ is the optical power, $\alpha$ is the optical absorption coefficient at the corresponding excitation wavelength $\lambda, V_{b i}$ is the built in potential of the diode, $\mathrm{V}$ is the external applied voltage and $\epsilon$ is the semiconductor dielectric constant. The total current $I_{\text {illum }}$ produced by the device is then the sum of $I_{\lambda}^{G}$ and $I_{\lambda}$, and thus, the responsivity $\mathrm{R}$ of the detector, according to equations 1 and 2 , can be written as:

$$
R=\frac{I_{\text {illum }}-I_{d}}{P}=\frac{\left(\exp \frac{\Delta \Phi_{b}}{k T}-1\right) I_{d}+I_{\lambda}}{P}
$$

According to equation 3, and as shown in table 1, the higher are the dark current and SBH decrease, the higher are $\mathrm{R}$, and thus the internal gain. Nevertheless, since a high dark current is generally a drawback for PDs, the SBH decrease has to be as large as possible and the dark current should be tuned to preserve large internal gain, since a too small value of the dark current leads to a low value of the internal gain even if the SBH decrease is large.

A large dark current is generally due to a high-density threading dislocations and high background carrier concentration. To reduce the dark current Sang et $\mathrm{al}^{3}$ have proposed the use of a $\mathrm{CaF}_{2}$ insulated layer deposited on InGaN material between the Schottky contact and the active layer, as shown on Fig.2. The dark current of the $\mathrm{PD}$ was reduced by six orders of magnitude compared with those without $\mathrm{CaF}_{2}$ layer, with a value of the internal gain equal to 40 . Within a less complex process, Lee et al ${ }^{18}$ have used a thin low-temperature GaN cap layer on top of an undoped GaN layer and below the Schottky contact, as reported on Fig. 3. In this case, the dark current of the PD was reduced by four orders of magnitude compared with those without cap layer, but without any internal gain.

In this paper, instead of using the approach of insulation layer between the Schottky contact and the active layer, we aim to develop large internal gain MSM PD with reduced dark current based on GaN-type active layer with low electrical conductivity. To achieve this purpose, three types of active layers are studied. The first one 
Table 1. Room temperature values of dark current $I_{d}$ (at $\mathrm{V}=10 \mathrm{~V}$ ), SBH reduction $\Delta \Phi_{b}$, photocurrent $I_{\lambda}^{G}$ and internal gains (at optical power in the $\mathrm{nW}$ range and $\mathrm{V}=10 \mathrm{~V}$ ) for different PDs. *this work

\begin{tabular}{cccccc} 
Material & PD type & $I_{d}$ & $\Delta \Phi_{b}$ & $I_{\lambda}^{G}$ & Gain \\
\hline $\mathrm{ZnO}^{1}$ & MSM & $15 \mathrm{~mA}$ & $52 \mathrm{meV}$ & $110 \mathrm{~mA}$ & $10^{4}$ \\
$\mathrm{MgZnO}^{9}$ & $\mathrm{MSM}$ & $100 \mu \mathrm{A}$ & $30 \mathrm{meV}$ & $317 \mu \mathrm{A}$ & 15 \\
$\mathrm{GaN}^{15}$ & MSM & $300 \mathrm{nA}$ & $70 \mathrm{meV}$ & $4.5 \mu \mathrm{A}$ & 50 \\
$\mathrm{SiC}^{16}$ & Schottky & $0.1 \mathrm{nA}$ & $300 \mathrm{meV}$ & $10 \mu \mathrm{A}$ & 2 \\
\hline $\mathrm{GaN}^{*}$ & $\mathrm{MSM}$ & $4.4 \mathrm{~mA}$ & $6 \mathrm{meV}$ & $5.5 \mathrm{~mA}$ & $10^{5}$ \\
$\mathrm{GaN}^{*} \mathrm{Fe}^{*}$ & $\mathrm{MSM}$ & $30 \mathrm{nA}$ & $50 \mathrm{meV}$ & $205 \mathrm{nA}$ & 15 \\
BGaN$^{*}$ & $\mathrm{MSM}$ & $5.4 \mu \mathrm{A}$ & $20 \mathrm{meV}$ & $11.7 \mu \mathrm{A}$ & $10^{3}$ \\
BGaN-SL$^{*}$ & $\mathrm{MSM}$ & $468 \mu \mathrm{A}$ & $16 \mathrm{meV}$ & $865 \mu \mathrm{A}$ & $3.5 \times 10^{4}$
\end{tabular}

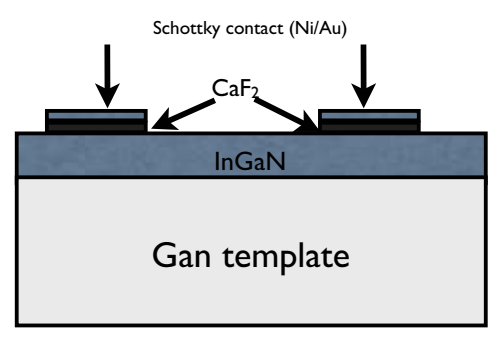

Figure 2. InGaN-based UV PD using a $\mathrm{CaF}_{2}$ layer as proposed by Sang et al. ${ }^{3}$

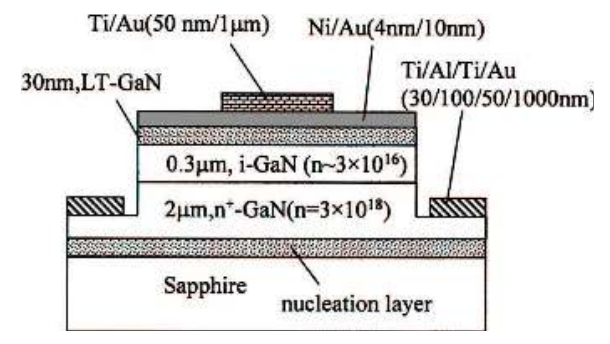

Figure 3. GaN-based UV PD using a thin lowtemperature GaN layer as proposed by Lee et al. ${ }^{3}$

consists in a $0.4 \mu \mathrm{m} \mathrm{BGaN} \mathrm{thick} \mathrm{monolayer} \mathrm{grown} \mathrm{on} \mathrm{GaN} \mathrm{template} \mathrm{with} \mathrm{several} \mathrm{boron} \mathrm{concentration.} \mathrm{The}$ second and third ones are superlattices (SL) structures of BGaN-GaN and BGaN-AlN, respectively. Indeed, as shown previously, ${ }^{19}$ the electrical conductivity of the BGaN alloy can be tuned over more than seven orders of magnitude using low boron incorporation concentration (less than 2\%). Furthermore, the boron incorporation allows to tune the band gap value, ${ }^{20}$ yielding a proper adjustment of the wavelength detection in the UV range (see Fig.1). Moreover, thanks to the boron incorporation, it is possible to use lattice engineering in order to reach a lattice match between the active layer and $\mathrm{SiC}$ and $\mathrm{Al}_{2} \mathrm{O}_{3}$ substrates. Finally, the performances of the new BGaN type-based PDs are compared with those of a $0.4 \mu \mathrm{m}$ thick GaN-based MSM UV PD.

\section{SAMPLES PREPARATION AND EXPERIMENTAL DETAILS}

The design of the samples used in this work are schematically shown in Fig.4. The first PD, which is the reference $\mathrm{PD}$, is based on a commercially available $400 \mathrm{~nm}$ thick undoped GaN grown on sapphire substrate. The first BGaN-based PD consists of BGaN monolayers with several boron incorporation rates varying from $0.05 \%$ up to $0.82 \%$ grown on GaN and AlN templates. The second and third BGaN-based PDs are SLs structures of BGaNGaN consisting of 10 pairs of $20 \mathrm{~nm}$ thick BGaN with $3.6 \%$ B and $20 \mathrm{~nm}$ thick GaN) from one part, and from the other part, of BGaN-AlN consisting of 10 pair of $2 \mathrm{~nm}$ thick BGaN with $20 \% \mathrm{~B}$ and $2 \mathrm{~nm}$ thick AlN) grown on GaN and AlN templates respectively. The corresponding doping concentrations $\mathrm{N}_{D}$, as deduced from $\mathrm{C}-\mathrm{V}$ measurements, range between $15 \times 10^{18} \mathrm{~cm}^{-3}$ and $2 \times 10^{15} \mathrm{~cm}^{-3}$ according to the B incorporation rate increase. All the BGaN structures (monolayers and SLs) were grown by MOVPE using a T-shape reactor. TMG, TEB and $\mathrm{NH}_{3}$ were used as source for gallium, boron, and nitrogen, respectively. Details on the growth conditions, boron incorporation, and layers morphology are mentioned in previous works. ${ }^{20,21}$ All the samples were unintentionally doped. The samples surface was chemically treated before the deposition of Pt, using standard photolithography process, to form interdigital electrodes with $10 \mu \mathrm{m}$ finger width, $300 \mu \mathrm{m}$ finger length, and $10 \mu \mathrm{m}$ finger gap as shown on figure 4. Current-voltage measurements were performed using a Keithley 2602 source measurement unit. The spectral response was carried out using a $100 \mathrm{~W}$ xenon arc lamp, a monochromator and a lock-in 


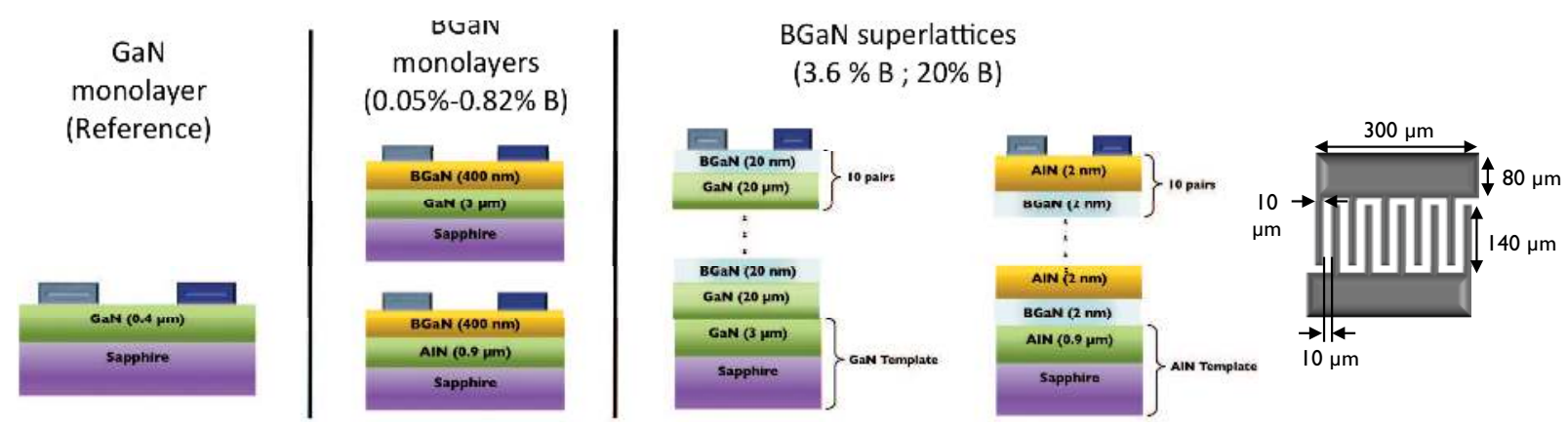

Figure 4. Design of the different PD structures and interdigitated electrode structure used in this work

amplifier with an optical chopper. The voltage and time dependencies of the responsivity were recorded by a 500 MHz-bandwidth digital oscilloscope and a $266 \mathrm{~nm} \mathrm{Nd:YAG} \mathrm{laser} \mathrm{(0.1} \mathrm{ns} \mathrm{pulse} \mathrm{width,} 10 \mathrm{kHz}$ repetition rate).

\section{RESULTS AND DISCUSSION}

\subsection{Structural and morphological characterizations}

SIMS analysis has been carried out on the different samples. As an example, Fig.5 shows the concentration of boron and gallium as a function of the layer depth obtained on both BGaN monolayer $(0.7 \% \mathrm{~B})$ and $\mathrm{BGaN}(3.6 \% \mathrm{~B})$-GaN SL. In the monolayer, the profile of boron concentration is flat, indicating the excellent distribution of boron along the growth direction. In both monolayer and SL, the anti-phase variation of the boron profile with respect to the gallium underlines that the boron element sits well in the Ga site as expected for a III-III-V alloy. AFM, SEM, XRD and HAADF-STEM analyses have been also carried out. In all samples, a columnar structure is observed, each column consisting of a grain of about $100 \mathrm{~nm}$ in width. The different BGaN layers are relatively homogeneous suggesting that there is no compositional inhomogeneity in the layers. Numerous stacking faults were also identified. The crystalline quality was good enough to observe room temperature photoluminescence.
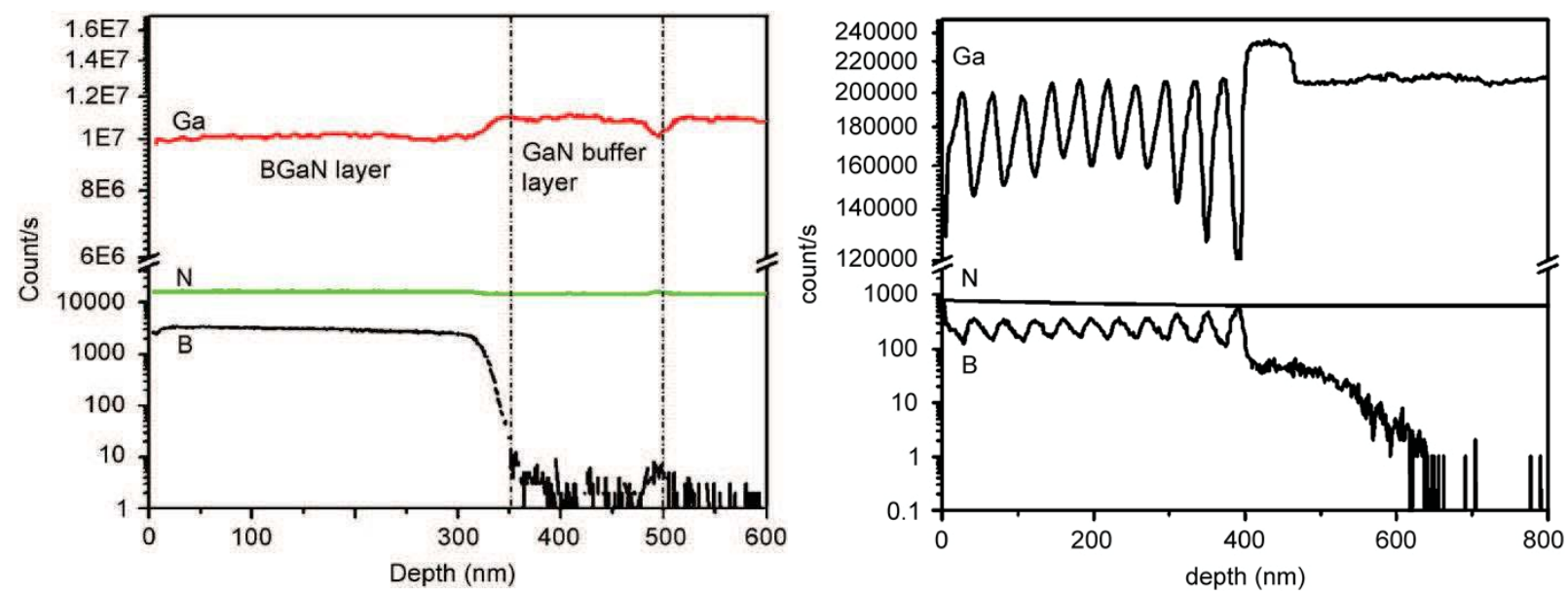

Figure 5. SIMS profiles of both BGaN monolayer $(0.7 \%$ B) and BGaN(3.6\%B)-GaN SL

\subsection{Electrical characterizations}

Fig.6 shows the I-V characteristics for the whole set of BGaN devices measured in dark condition. A symmetrical rectifying behavior is obtained, indicating back-to-back Schottky contacts. With the increase of the boron 
concentration and thus large increase of the layer resistivity, the dark current in the BGaN monolayers decreases, as expected, down to a few $\mathrm{nA}$ for a $\mathrm{B}$ concentration of $0.82 \%$. In the case of the SL structures, the BGaN-AlN SL-based PD exhibits very low dark current (few pA) while the BGaN-GaN SL-based PD shows a relatively high value of the dark current (few hundreds of $\mu \mathrm{A}$ ) although the high $\mathrm{B}$ concentration (3.6\%). This probably originates from the GaN interlayers, which, in contrary to AlN interlayers, possess low value of resistivity and thus allow large current flow. The Schottky barriers heights for the whole set of structures have been estimated

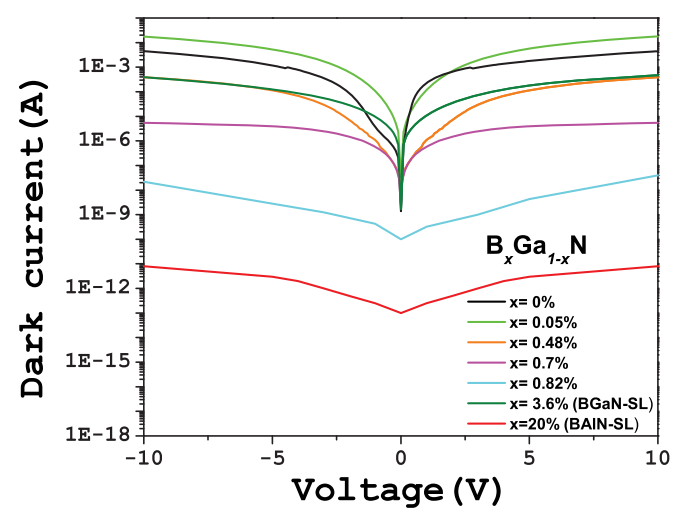

Figure 6. I-V characteristics for the whole set of BGaN devices measured in dark condition

using I-V measurements on Schottky diodes and the thermionic emission model, and ranges between $0.74 \mathrm{eV}$ and $0.52 \mathrm{eV}$ for the different structures under test.

\subsection{Voltage, wavelength, and optical power dependence of the responsivity}

Fig.7 shows the voltage dependence of R obtained, for the MSM PDs, at $\lambda=266 \mathrm{~nm}$ corresponding to a photon energy well above the band gap of the different PDs, except in the case of the BGaN-AlN SL structure, and for an optical power of $56 \mu \mathrm{W}$. In contrary to the BGaN(0.82\%)- and BGaN-AlN SL-based PDs for which no

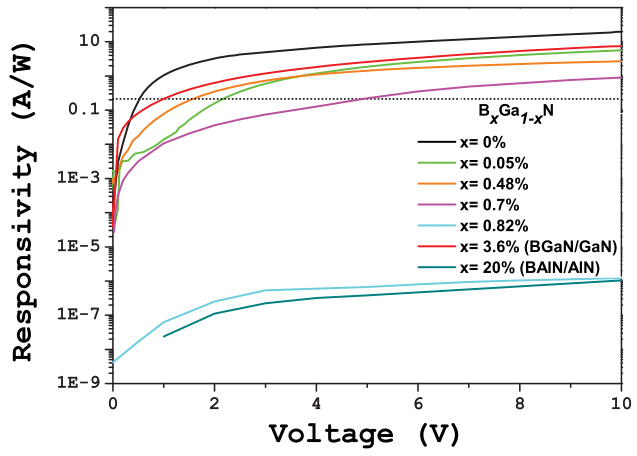

Figure 7. Voltage dependence of the responsivity of the different MSM PDs at $\lambda=266 \mathrm{~nm}$ and $\mathrm{P}=56 \mu \mathrm{W}$. The dashed line corresponds to the theoretical value of $\mathrm{R}$ for $100 \%$ of QE.

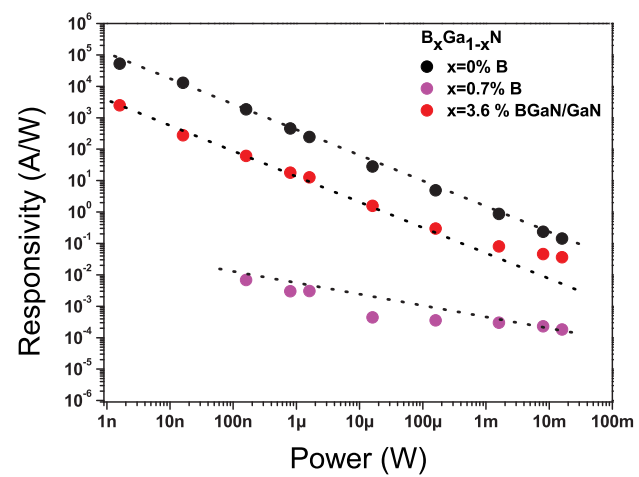

Figure 8. Optical power dependence of the responsivity of three type of PDs measured at $\lambda=355 \mathrm{~nm}$.

internal gain is observed whatever the bias voltage, all the BGaN-, BGaN-GaN SL- and GaN-based PDs exhibit 
internal gain for applied voltages above $5 \mathrm{~V}(0.7 \% \mathrm{~B}), 2 \mathrm{~V}((0.05 \% \mathrm{~B}), 1.5 \mathrm{~V}(0.48 \%), 1 \mathrm{~V}(\mathrm{BGaN}-\mathrm{GaN})$, and $0.5 \mathrm{~V}$ $(\mathrm{GaN})$. The variation of $\mathrm{R}$ from one $\mathrm{PD}$ to the other is closely related to the value of the dark current, which mainly controls the internal gain, since the SBH decrease $\Delta \Phi_{b}$, derived, using equation 3, from the data of Fig.7, and reported in table 3.4, has the same order of magnitude in the different PDs. It is to be noted that with the decreasing optical power $\mathrm{P}$ (as reported in Fig.8 for three types of PDs), R increases for each PD as $\mathrm{P}^{\frac{1}{2}}$ relationship. This relationship suggests ${ }^{17}$ the existence of traps in the band gap acting as recombination centers with an acceptor character, supporting the explanation of the existence of internal gain described above. Thus, when operating at lower optical power (nW range), the internal gain, of GaN- and BGaN-GaN SL-based PDs for instance, which is equal to 100 and 36, respectively, for an optical power of $56 \mu \mathrm{W}$ (case of the data plotted in Fig.7), becomes very high and reaches values equal to $10^{5}$ and $3 \times 10^{4}$, respectively.

The normalized spectral responsivity of the PDs (except the BGaN-AlN SL one) measured under a bias of 10V is shown in Fig.9. The wavelength cutoff, corresponding to the value of the wavelength at which the responsivity is maximum, is found to be equal to $360 \mathrm{~nm}$ for the GaN-based PD, and increases to values equal to $361 \mathrm{~nm}(0.05 \%$ B), $367 \mathrm{~nm}(0.48 \% \mathrm{~B}), 373 \mathrm{~nm}(0.7 \% \mathrm{~B})$, and $380 \mathrm{~nm}(0.82 \% \mathrm{~B})$ for the BGaN-based PDs, respectively. This red-

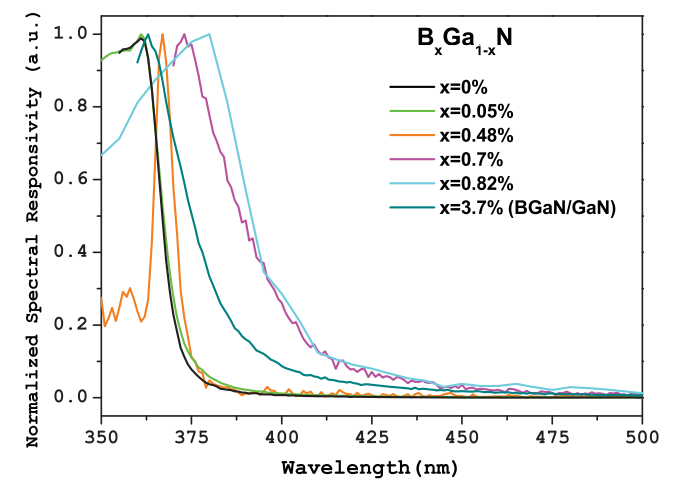

Figure 9. Normalized spectral responsivity for the different UV PDs under a bias of $10 \mathrm{~V}$

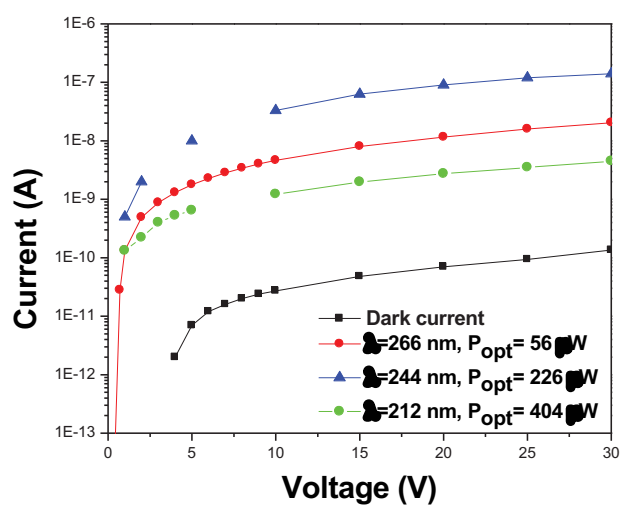

Figure 10. I-V characteristics of the BGaN-AlN SL-based PD measured under different UV radiations

shift of the wavelength cutoff is due, as expected, to the band gap bowing induced by the boron incorporation. ${ }^{22}$ In the case of the BGaN-GaN SL-based PD, and according to this band gap bowing, a wavelength cutoff equal to $387 \mathrm{~nm}$ should be observed. In contrary, the wavelength cutoff is found to be equal to $365 \mathrm{~nm}$, very close to the one of the GaN-based PD. This discrepancy could probably be attributed to the extrapolation procedure for the calculation of the wavelength cutoff for BGaN materials with B composition equal to 3.6\%, which is based on a value of the bowing coefficient determined according to band gap measurements in BGaN materials for B composition up to only $1.8 \% .^{22}$ Further studies need to be performed to clarify this point. It is also to be noted that the decrease of $\mathrm{R}$ with increasing wavelength above the cutoff is less sharp for both BGaN- and BGaN-GaN SL-based PDs than for the GaN-based PDs. This is probably due to the higher dislocation density existing in the $\mathrm{BGaN}$ material. Due to the same reason, the value of the UV/VIS rejection ratio is more than one order of magnitude higher for the GaN based PD $\left(2 \times 10^{3}\right)$ than for the BGaN- and BGaN-GaN SL-based PDs.

Since the wavelength cutoff of the xenon arc lamp is equal to $350 \mathrm{~nm}$, in order to determine the cutoff wavelength of the BGaN-AlN SL-based PD, the photocurrent under 212nm, 244nm, and $266 \mathrm{~nm}$ laser irradiations have been measured and reported in Fig.10. Unfortunately, the data of Fig.10 do not allow any accurate conclusions to be drawn and only indicate that the wavelength corresponding to the maximum value of the photocurrent ranges between $212 \mathrm{~nm}$ and $266 \mathrm{~nm}$. Additional measurements for intermediate wavelengths are needed to clarify this point. 


\subsection{Time dependence of the PDs photocurrent responses}

The records of the photocurrent transients of three PDs, at $\lambda=266 \mathrm{~nm}, \mathrm{~V}=10 \mathrm{~V}$, and for optical pulse duration of $0.1 \mathrm{~ns}$ ( $11 \mathrm{~W}$ of optical peak power) and several tens of seconds (56 $\mu \mathrm{W}$ of optical peak power), respectively, are shown in Fig.11. The values of the $10 \%$ to $90 \%$ rise and fall times as well as those of the peak responsivity, which can be deduced from the data of Fig.11, are reported in table 3.4. At high optical power, all PDs exhibit low values of the rise time (less than $15 \mathrm{~ns}$ ). BGaN-GaN SL-based PDs exhibit relatively low photocurrent time-decays ( $90 \%$ of the response is reached after $45 \mathrm{~ns}$ and $15 \mathrm{~ns}$, respectively), whereas more than hundred times higher time-decays are observed in the GaN- and BGaN monolayer-based PDs (90\% of the response is reached after $15 \mu \mathrm{s}$ and $6.7 \mu \mathrm{s}$, respectively). These relatively slow time-decays (the initial current value before illumination is recovered within less than $100 \mu$ s after the optical power is turned off) can be attributed to a persistent photoconductivity effect caused by trapping/detrapping processes of photo-generated carriers at deep level defects in the band gap of the material. ${ }^{5}$ As it has been discussed above, at such high optical power, much lower internal gain is expected and $\mathrm{R}$ is in the $\mathrm{mA} / \mathrm{W}$ range for all PDs. The highest value of $\mathrm{R}$, equal to $30 \mathrm{~mA} / \mathrm{W}$, is obtained in the BGaN-GaN SL-based PD, and is $25 \%$, and $710 \%$ higher than those of the GaN-, and BGaN monolayer-based PD, respectively. The low value of $\mathrm{R}$ for the BGaN monolayer-based PD can be explained by the relatively poor structural quality of the $400 \mathrm{~nm}$ thick BGaN monolayer, ${ }^{21}$ yielding low values of the photo-carrier mobility and diffusion length. In contrary, the higher structural quality of the $20 \mathrm{~nm}$ thick BGaN layers ${ }^{21}$ in the BGaN-GaN SL-based PD leads to higher values of the photo-carrier mobility and diffusion length and, thus, responsivity. BGaN-GaN SL- and GaN-based PDs exhibit relatively low photocurrent time-decays (see Fig.11), whereas more than hundred times higher time-decays are observed in the GaN- and BGaN-based PDs. At low optical power (see Fig.12), and thus higher internal gain, for all PDs, R is in the A/W range and the rise times and recovering times of the initial current value are much longer and reach several tens of $\mathrm{ms}$ and seconds, respectively, as reported in table 3.4 .

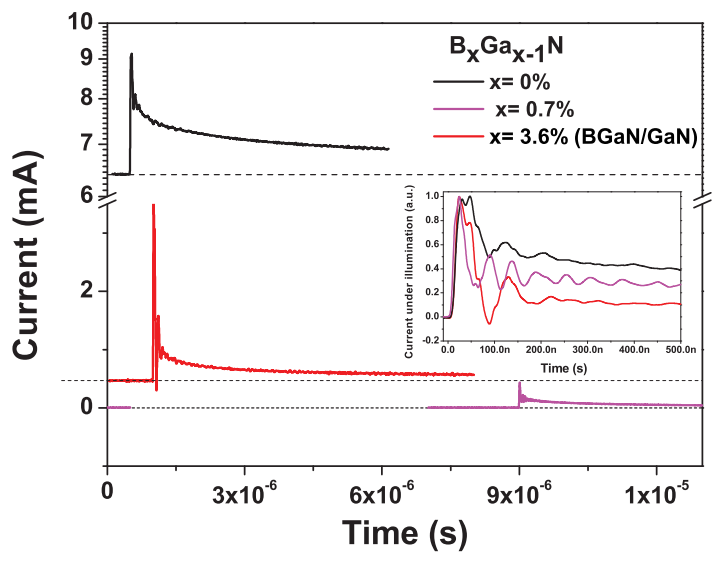

Figure 11. Time dependence of the photocurrent in the three MSM PDs recorded at $\lambda=266 \mathrm{~nm}$, for $\mathrm{V}=10 \mathrm{~V}$, and for an optical pulse duration of $0.1 \mathrm{~ns}$ with a peak power of $11 \mathrm{~W}$

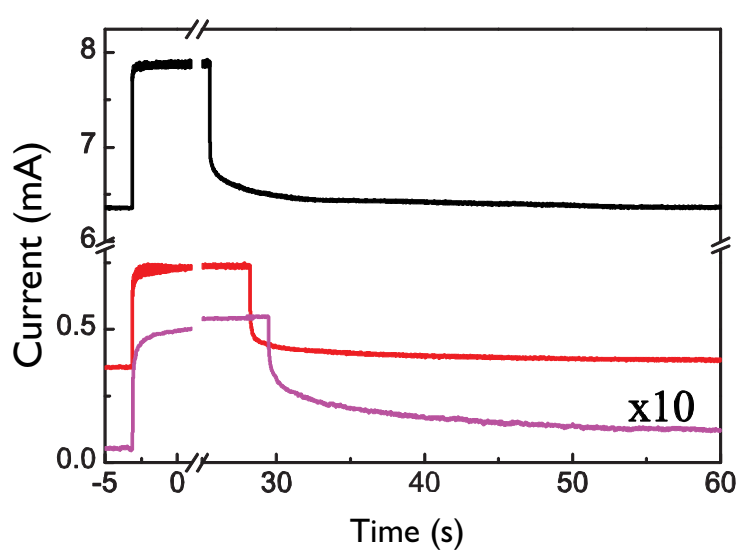

Figure 12. Time dependence of the photocurrent for an optical pulse duration of several tens of seconds with a peak power of $56 \mu \mathrm{W}$. Same legend as Fig.11

\section{CONCLUSION}

To summarize our work, Fig.13 shows the B concentration dependence of the dark current density (red dots), Schottky barrier decrease (black dots) and low optical power gain (gray line) in BGaN monolayer-based and BGaN SL-based UV photodetectors. In the case of the BGaN monolayer-based PD, the B concentration increase leads to the increase of the active layer resistivity and thus to the decrease of the dark current density. As the BGaN monolayers are thick $(400 \mathrm{~nm})$, they exhibit a high-density threading dislocations leading to a large 
Table 2. Values of the responsivity $\mathrm{R}$ (after a correction taking into account of 40dB decrease in the photocurrent response due to the limitation of the measurements system), $10 \%$ to $90 \%$ rise $\tau_{r}$ and fall $\tau_{f}$ times of the different PDs, measured at $\lambda=266 \mathrm{~nm}$ and $\mathrm{V}=10 \mathrm{~V}$, for low $(56 \mu \mathrm{W})$ and high $(11 \mathrm{~W})$ optical power.

\begin{tabular}{ccccccc} 
& \multicolumn{3}{c}{ High optical power } & \multicolumn{3}{c}{ Low optical power } \\
\hline Material & $\mathrm{R}(\mathrm{A} / \mathrm{W})$ & $\tau_{r}$ & $\tau_{f}$ & $\mathrm{R}(\mathrm{A} / \mathrm{W})$ & $\tau_{r}$ & $\tau_{f}$ \\
\hline GaN & 0.024 & $15 \mathrm{~ns}$ & $15 \mu \mathrm{s}$ & 19.2 & $42 \mathrm{~ms}$ & $4 \mathrm{~s}$ \\
BGaN & 0.0037 & $15 \mathrm{~ns}$ & $6.7 \mu \mathrm{s}$ & 0.9 & $4 \mathrm{~s}$ & $60 \mathrm{~s}$ \\
BGaN-SL & 0.03 & $15 \mathrm{~ns}$ & $44 \mathrm{~ns}$ & 7.4 & $0.56 \mathrm{~s}$ & $20 \mathrm{~s}$
\end{tabular}

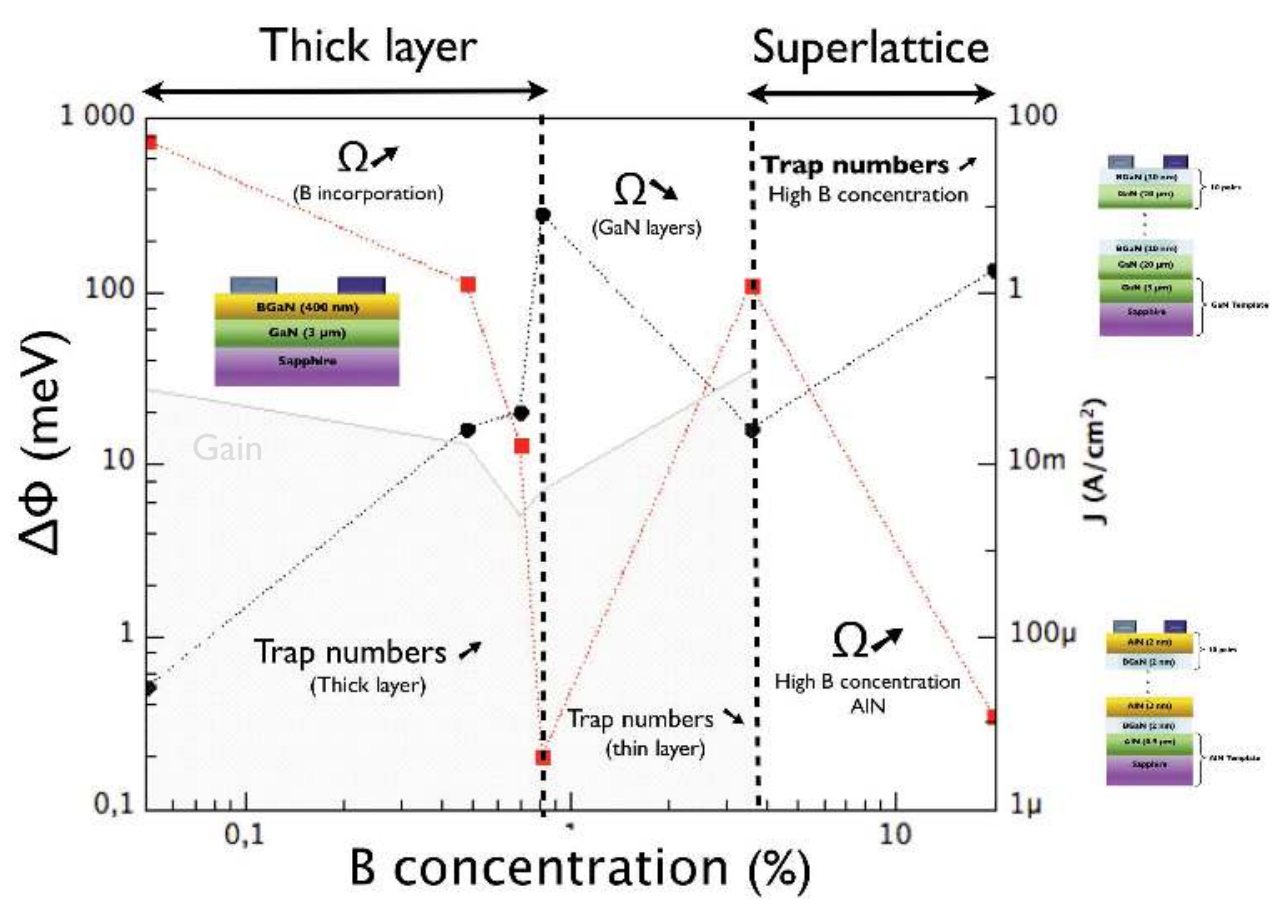

Figure 13. B concentration dependence of the dark current density (red dots), Schottky barrier (black dots) and $\mu \mathrm{W}$ range optical power $(\lambda=266 \mathrm{~nm})$ gain (gray line) in BGaN monolayer-based and BGaN SL-based UV photodetectors

number of trapping states at the active layer-metal interface, and thus to an enhancement of the SBH decrease under illumination yielding large internal gain PD if the dark current is not too weak. In the case of BGaN-GaN SL-based PD, even for large B concentration, due to leakage current in the GaN interlayers, the dark current density is higher than the one of BGaN monolayer-based PD. As the BGaN monolayers in the SL structure is thin $(20 \mathrm{~nm})$, they exhibit a lower-density threading dislocations leading to a lower number of trapping states at the active layer-metal interface, and thus to a decrease of the SBH decrease under illumination yielding large internal gain PD since the dark current is relatively large. In the case of BGaN-AlN SL-based PD, the large B concentration, and the high resistivity of the AlN interlayers, leads to a very weak dark current density. Even if the BGaN monolayers in the SL structure is very thin $(2 \mathrm{~nm})$, the large B concentration yields a high-density threading dislocations leading to a large number of trapping states at the active layer-metal interface, and thus to an enhancement of the SBH decrease under illumination yielding large internal gain PD if the dark current is not too weak. It is to be noted that for this last structure, the value of the SBH decrease under illumination has been obtained at a wavelength of $266 \mathrm{~nm}$ far away from the maximum absorption of the structure.

In conclusion, MSM solar-blind ultraviolet PDs were fabricated using quasi-alloy of BGaN/GaN SLs as the 
active layer. The boron incorporation rate allows to decrease the dark current by one order of magnitude compared to GaN-based PD, and to tune the cut-off wavelength of the PD while maintaining high DC internal gain (up to $3 \times 10^{4}$ for optical power in the $\mathrm{nW}$ range). In the high optical power regime, more than two orders of magnitude lower time response and 25\% higher responsivity than GaN-based PD have been obtained. The improvement of the UV/VIS rejection ratio and sharpness of the wavelength cutoff is in progress and relies on the optimization of the crystal quality of the active layers which could be obtained by the growth of thinner layers of the BGaN material.

\section{ACKNOWLEDGMENTS}

This work was supported by both the French National Center of Scientific Research (CNRS) within a PEPS research program, and the Region Lorraine in the frame of a local program. The authors would like to express their gratitude to Jeramy Dickerson for his help on spectral responsivity measurements

\section{REFERENCES}

[1] Liu, J., Shan, C., Li, B., Zhang, Z., Yang, C., Shen, D., and Fan, K., "High responsitivity ultraviolet photodetector realized via a carrier-trapping process," Appl. Phys. Lett. 97, 251102 (2010).

[2] Turgut, T., Tolga, Y., Erkin, U., and Ozbay, O. E., "Solar-blind algan-based p-i-n photodetectors with high breakdown voltage and detectivity," Appl. Phys. Lett 92, 103502 (2008).

[3] Sang, L., Liao, M., Koide, Y., and Sumiya, M., "High-performance metal-semiconductor-metal ingan photodetectors using caf2 as the insulator," Appl. Phys. Lett. 98, 103502 (2001).

[4] Sang, L., Liao, M., Koide, Y., and Sumiya, M., "High-temperature ultravioler detection based on ingan schottky photodiodes," Appl. Phys. Lett. 99, 031115 (2011).

[5] Martens, M., Schlegen, J., Vogt, P., Brunner, F., Lossy, R., Wurfi, J., Weyers, M., and Kneissl, M., "High gain ultraviolet photodetectors based on algan/gan heterostructures for optical switching," Appl. Phys. Lett. 98, 211114 (2001).

[6] Malinowski, P., Duboz, J., Moor, P. D., Minoglou, K., John, J., Horcajo, S., Semond, F., Frayssinet, E., Verhoeve, P., Esposito, M., Giordanengo, B., BenMoussa, A., Mertens, R., and Hoof, C. V., "Extreme ultraviolet detection using algan-on-si inverted schottky photodiodes," Appl. Phys. Lett. 98, 141104 (2011).

[7] Li, J., Zhao, M., and Wang, X., "High performance schottky uv photodetectors based on epitaxial algan thin film," Physica B 405, 996 (2010).

[8] Barkad, H., Soltani, A., Mattallah, M., Gerbedoen, J., Rousseau, M., Jaeger, J. D., Moussa, A. B., Mortet, V., Haenen, K., Benbakhti, B., Moreau, M., Dupuis, R., and Ougazzaden, A., "Design, fabrication and physical analysis of tin/aln deep uv photodiodes," J. Phys. D 43, 465104 (2010).

[9] Zhang, Z., Wenckstern, H., Schmidt, M., and Grundmann, M., "Wavelength selective metal-semiconductormetal photodetectors based on (mg,zn)o-heterostructures," Appl. Phys. Lett. 99, 083502 (2011).

[10] Hou, Y., Mei, Z., Liu, Z., Zhang, T., and Du, X., "Mg0.55zn0.45o solar-blind ultraviolet detector with high photoresponse performance and large internal gain," Appl. Phys. Lett. 98, 103506 (2011).

[11] Tabares, G., Hierro, A., Ulloa, J., Guzman, A., Munoz, E., Nakamura, A., Hayashi, T., and Temmyo, J., "High responsitivity and internal gain mechanisms in au-znmgo schottky photodiodes," Appl. Phys. Lett. 96, $101112(2010)$.

[12] Zhu, H., Shan, C., Wang, L., Zheng, J., Zhang, J., Yao, B., and Shen, D., "Metal-oxide-semiconductorstructured mgzno ultraviolet photodetector with high internal gain," J. Phys. Chem. C 114, 7169 (2010).

[13] Shinde, S. and Rajpure, K., "Fast response ultraviolet ga-doped zno based photoconductive detector," Mat. Res. Bull. 46, 1734 (2011).

[14] Kosyachenko, L., Lashkarev, G., Sklyarchuk, V., Ievtushenko, A., Sklyarchuk, O., Lazorenko, V., and Ulyashin, A., "Zno-based photodetector with photocurrent gain," Phys. Stat. Sol. A 207(8), 1972 (2010).

[15] Katz, O., Garber, V., Meyler, B., Bahir, G., and Salzman, J., "Gain mechanism in gan schottky ultraviolet detectors," Appl. Phys. Lett. 79(10), 1417 (2001). 
[16] Sciuto, A., Roccaforte, F., Franco, S. D., Raineri, V., Billotta, S., and Bonanno, G., "Photocurrent gain in 4h-sic interdigit schottky uv photodetectors with a thermally grown oxyde layer," Appl. Phys. Lett. 90, 223507 (2007).

[17] Zhang, S., Wang, W., Shtau, I., Yun, F., He, L., Morkoc, H., Zhou, X., Tamargo, M., and Alfano, R., "Backilluminated gan/algan heterojunction ultraviolet photodetector with high internal gain," Appl. Phys. Lett. 81, 4862 (2002).

[18] Lee, M., Sheu, J., Lai, W., Chang, S., Su, Y., Chen, M., Kao, C., Chi, G., and Tsai, J., "Gan schottky barrier photodetectors with a low-temperature gan cap layer," Appl. Phys. Lett. 82, 2913 (2003).

[19] Baghdadli, T., Hamady, S. O. S., Gautier, S., Moudakir, T., Benyoucef, B., and Ougazzaden, A., "Electrical and structural characterizations of bgan thin films grown by metal-organic vapor-phase epitaxy," Phys. Stat. Sol. C 6(S2), S1029 (2009).

[20] Gautier, S., Sartel, C., Hamady, S. O. S., Maloufi, N., Martin, J., Jomard, F., and Ougazzaden, A., "Movpe growth study of bgan on gan template substrate," Superlatt. Microstruct. 40, 233 (2006).

[21] Ougazzaden, A., Gautier, S., Sartel, C., Maloufi, N., Martin, J., and Jomard, F., "Bgan materials on gan/sapphire substrate by movpe using n2 carrier gas," J. Cryst. Growth 298, 316 (2007).

[22] Ougazzaden, A., Gautier, S., Moudakir, T., Djebbour, Z., Lochner, Z., Choi, S., Kim, H., Ryou, J.-H., Dupuis, R., and Sirenko, A., "Bandgap bowing in bgan thin films," Appl. Phys. Lett. 93, 083118 (2008). 